\title{
Enabling Serendipitous Search on the Web of Data Using Prolog
}

\author{
Jan Wielemaker \\ University of Amsterdam
}

\begin{abstract}
The Web of Data, also called the Semantic Web, provides a knowledge representation formalism based on a uniform triple-model: subject, predicate, object. A number of more expressive formalisms (e.g., RDFS, OWL, SKOS) are layered on top of the core triple-model. The Web of Data has been developed to represent machine readable knowledge on the internet. Designed to deal with heterogeneous knowledge, the technology underlying the Web of Data is also suitable to unify databases. We use this technology to unify collection information from multiple museums, based on diverse schemas and multiple controlled lists of terms (vocabularies). The resulting knowledge-base is enriched using automatic discovery of mappings between vocabularies. The current challenge is how to provide meaningful services for the end-user based on this knowledge, in particular, how to provide meaningful semantically enriched search?

This tutorial presents the key-components of the Prolog-based ClioPatria toolkit and shows how this infrastructure can be used to explore the opportunities of semantic search. Topics discussed are: reasoning with and editing of RDF models, web-application programming in Prolog combined with AJAX technology and issues when using Prolog for programming 'at large'. Prolog is both an RDF query language and a general purpose programming language, and therefore provides a perfect platform for a Semantic Web research and applications.
\end{abstract}

